\title{
VARIATIONAL INTEGRATORS OF MIXED ORDER FOR DYNAMICAL SYSTEMS WITH MULTIPLE TIME SCALES AND SPLIT POTENTIALS
}

\author{
Theresa Wenger $^{1}$, Sina Ober-Blöbaum ${ }^{2}$, and Sigrid Leyendecker ${ }^{1}$ \\ ${ }^{1}$ Chair of Applied Dynamics \\ University of Erlangen-Nuremberg \\ Haberstrasse 1, 91058 Erlangen, Germany \\ e-mail: theresa.wenger@fau.de, sigrid.leyendecker@fau.de \\ ${ }^{2}$ Department of Engineering Science \\ University of Oxford \\ Parks Road, Oxford, OX1 3PJ, United Kingdom \\ e-mail:sina.ober-blobaum@eng.ox.ac.uk
}

Keywords: multiple time scales, higher order variational integrators, Fermi-Pasta-Ulam problem

\begin{abstract}
The simulation of mechanical systems that act on multiple time scales, caused e.g. by different types or stiffnesses in potentials, is challenging as a stable integration of the fast dynamics requires a highly accurate approximation whereas for the simulation of the slow part a coarser approximation is accurate enough. With regard to the general goals of any numerical method, high accuracy and low computational costs, the presented variational integrators of mixed order couple coarse and fine approximations. The idea builds up on the higher order Galerkin variational integrators in [9] that are derived via Hamilton's variational principle with a polynomial to approximate the configuration and an appropriate quadrature formula for the approximation of the integral of the Lagrangian. For the variational integration of systems with dynamics on multiple time scales, we use polynomials of different degrees to approximate the components that act on different time scales. Furthermore, quadrature formulas of different order approximate the integrals of the single energy contributions of the Lagrangian. This approach provides great flexibility in the design of the integrators. Their performance is investigated numerically by means of the Fermi-Pasta-Ulam problem and a numerical analysis regarding accuracy versus efficiency is carried out, where we focus on the integrators most promising to resolve the mentioned trade-off.
\end{abstract}




\section{INTRODUCTION}

Many systems in Lagrangian mechanics exhibit dynamics on multiple time scales caused by different types and stiffnesses in potentials, e.g. highly oscillatory problems with a potential that can be split in a fast quadratic part and a slow nonlinear part. The fast dynamics require a highly accurate approximation whereas for the slow motion a coarser approximation is sufficient. This contrast leads to a conflict of the general goals, high accuracy and low computational costs, and thus is challenging for numerical methods. For the simulation of Lagrangian mechanical systems, variational integrators are convenient as they inherit specific properties of the underlying system by construction. The variational integrators base on a discrete version of Hamilton's principle inducing symplectic and momentum preserving integration schemes with excellent long time behaviour [8].

Multirate integrators resolve the mentioned trade-off by separating the degrees of freedom into fast and slow components and integrating them on different time grids, e.g. [5], [3], [1], [2]. A rather large time step is chosen for the integration of the slow components and a fine grid for the fast components. In [4], multirate schemes that base on partitioned Runge-Kutta methods are introduced. The multirate integrators in [7] base on variational mechanics allowing flexibility in design as the use of different quadrature rules for the approximation of the appearing integrals is enabled. Another approach is the splitting method that does not tend to actually resolve the fast motion, but to capture the multiscale phenomena of slow energy exchange and adiabatic invariants, e.g. implicit-explicit (IMEX) methods. The IMEX methods integrate the expensive slow forces explicitly and the cheap fast forces implicitly, see e.g. [5], [6]. A derivation of the IMEX numerical integration from a Lagrangian variational point of view is given in [10]. Another efficient framework for simulating systems with dynamics on multiple time scales is provided by averaging methods. The fast motion is not resolved, only an estimation is fed into the slow equations of motion. A powerful concept is known as HMM (heterogeneous multiscale methods [12]) with a wide range of applications in multiscale and multiphysics problems. Based on a top-down strategy, the motion of the microscale model is estimated through averaging and supplies the incomplete macroscale model with the missing information. Another example of averaging methods are FLAVORS (flow averaging integrators [11]). These structure preserving integrators base on variational methods and use a splitting and resynchronisation technique to average the flow.

However, the idea of the presented integrators here is different. The underlying approach originates from the higher order Galerkin variational integrator in [9], where a polynomial is used to approximate the configuration together with an appropriate quadrature formula to approximate the integral of the Lagrangian. Let the configuration be split in components acting on various time-scales. Instead of multiple time grids, only one time grid is used and the components that act on different time scales are approximated via polynomials of different degrees. For the approximation of the integral of the Lagrangian, different quadrature formulas for its contributions (the kinetic energy, the slow and the fast potential) are considered. This yields a high number of possibilities to construct the variational integrators. With regard to save computational runtime, low degree polynomials to approximate the slow configuration and quadrature formulas of low order for the approximation of the slow integral are of particular interest as the former has a direct influence on the number of unknowns and the latter on the number of costly function evaluations of the slow potential in one time step. Higher accuracy, however, can be achieved by increasing the degree of the polynomial approximating the fast configuration. The performance and the computational efficiency of the variational integrators of mixed order are investigated 
numerically by means of a standard benchmark problem, the Fermi-Pasta-Ulam problem.

\section{VARIATIONAL INTEGRATORS OF MIXED ORDER}

\subsection{Lagrangian dynamics}

Consider an $n$-dimensional mechanical system on a configuration manifold $Q \subseteq \mathbb{R}^{n}$ with configuration vector $q(t) \in Q$ and velocity vector $\dot{q}(t) \in T_{q(t)} Q$ in the tangent space. The variable $t$ denotes the time bounded on the interval $[0, T] \subset \mathbb{R}$. The Lagrangian $L: T Q \rightarrow \mathbb{R}$ of the mechanical system consists of the difference of the kinetic energy $T(\dot{q})$ and the potential $U(q)$. Let the potential $U=V+W$ be composed of different parts with strongly varying dynamics acting on different time scales such that it can be split in a slow potential $V$ and a fast potential $W$. Furthermore, suppose that the $n$-dimensional variable $q$ can be separated into $n^{s}$ slow variables $q^{s} \in Q^{s}$ and $n^{f}$ fast variables $q^{f} \in Q^{f}$ such that $Q^{s} \times Q^{f}=Q$ and $q=\left(q^{s}, q^{f}\right)$ with $n^{s}+n^{f}=n$. It is assumed that the fast potential $W=W\left(q^{f}\right)$ depends on the fast degrees of freedom only whereas the slow potential depends on the complete configuration variable $q$, i.e. $V=V(q)$. Under these assumptions the Lagrangian $L: T Q \rightarrow \mathbb{R}$ reads

$$
L(q, \dot{q})=T(\dot{q})-V(q)-W\left(q^{f}\right)
$$

The action $S: C^{2}\left([0, T], Q^{s} \times Q^{f}\right) \rightarrow \mathbb{R}$ is the integral in $[0, T]$ of the Lagrangian

$$
S(q)=\int_{0}^{T} L(q, \dot{q}) d t
$$

depending on the smooth curves $q^{s} \in C^{2}\left([0, T], Q^{s}\right)$ and $q^{f} \in C^{2}\left([0, T], Q^{f}\right)$ with fixed initial value $q(0)=\left(q^{s}(0), q^{f}(0)\right)$ and fixed final value $q(T)=\left(q^{s}(T), q^{f}(T)\right)$. Hamilton's principle requires that the first variation of the action $S$ vanishes and yields the Euler-Lagrange equations of motion

$$
\begin{array}{r}
\frac{d}{d t} \frac{\partial T}{\partial \dot{q}^{s}}+\frac{\partial V}{\partial q^{s}}=0 \\
\frac{d}{d t} \frac{\partial T}{\partial \dot{q}^{f}}+\frac{\partial V}{\partial q^{f}}+\frac{\partial W}{\partial q^{f}}=0
\end{array}
$$

The Legendre-transform $\mathbb{F} L: T Q \rightarrow T^{*} Q$ reading in coordinate form

$$
\mathbb{F} L(q, \dot{q})=(q, p)=\left(q, \frac{\partial L}{\partial \dot{q}}(q, \dot{q})\right)
$$

relates $(q, \dot{q}) \in T Q$ to $(q, p) \in T^{*} Q$ in the cotangent space, where $p \in T_{q}^{*} Q$ denotes the conjugate momentum.

\subsection{Discrete variational principle}

The variational integrators of mixed order base on the higher order Galerkin variational integrators presented in [9], where a polynomial is used to approximate the continuous curve $q$ on one time step and the integral of the Lagrangian is approximated via an appropriate quadrature formula. The approach here allows for the dynamics acting on different time scales. For the approximation of the slow variables $q^{s}$ and the fast variables $q^{f}$, polynomials of different degrees are considered. With the splitting of the potential in a fast and a slow one different quadrature 
formulas for the approximation of each can be used.

The space of trajectories $\mathcal{C}\left([0, h], Q^{s}\right)=\left\{q^{s}:[0, h] \rightarrow Q^{s} \mid q^{s}(0)=q_{k}^{0, s}, q^{s}(h)=q_{k+1}^{0, s}\right\}$ and $\mathcal{C}\left([0, h], Q^{f}\right)=\left\{q^{f}:[0, h] \rightarrow Q^{f} \mid q^{f}(0)=q_{k}^{0, f}, q^{f}(h)=q_{k+1}^{0, f}\right\}$ reduces to the finitedimensional space of trajectories $\mathcal{C}^{p s}\left([0, h], Q^{s}\right)=\left\{q^{s} \in \mathcal{C}\left([0, h], Q^{s}\right) \mid q^{s} \in \Pi^{p s}\right\}$ and $\mathcal{C}^{p f}\left([0, h], Q^{f}\right)=\left\{q^{f} \in \mathcal{C}\left([0, h], Q^{f}\right) \mid q^{f} \in \Pi^{p f}\right\}$, whereby $\Pi^{p s}$ and $\Pi^{p f}$ denote the space of polynomials of degree ps respectively $p f$. With $p s+1$ configurations $q_{k}^{s}=\left(q_{k}^{0, s}, \ldots, q_{k}^{p s, s}\right) \in\left(Q^{s}\right)^{p s+1}$ at $p s+1$ control points $0=d_{0}^{s}<d_{1}^{s}<\ldots<d_{p s-1}^{s}<d_{p s}^{s}=1$, the $p s$ degree polynomial $q_{d}^{s}$ is uniquely defined such that it passes through each $q_{k}^{\nu, s}$ at the time $d_{\nu}^{s} h, \nu=0, \ldots, p s$. The polynomial $q_{d}^{f}$ of degree $p f$ is uniquely defined by the $p f+1$ configurations $q_{k}^{f}=\left(q_{k}^{0, f}, \ldots, q_{k}^{p f, f}\right) \in\left(Q^{f}\right)^{p f+1}$ at $p f+1$ control points $0=d_{0}^{f}<d_{1}^{f}<\ldots<$ $d_{p f-1}^{f}<d_{p f}^{f}=1$ passing through each $q_{k}^{\nu, f}$ at the time $d_{\nu}^{f} h, \nu=0, \ldots, p f$. Using the Lagrange polynomials $l_{\nu, p s}:[0,1] \rightarrow \mathbb{R}, \nu=0, \ldots, p s$ respectively $l_{\nu, p f}:[0,1] \rightarrow \mathbb{R}, \nu=0, \ldots, p f$, the polynomial $q_{d}^{s}$ approximating the slow variables and the polynomial $q_{d}^{f}$ approximating the fast variables on $[0, h]$ can be written in the form

$$
q_{d}^{s}\left(t ; q_{k}^{s}, h\right)=\sum_{\nu=0}^{p s} q_{k}^{\nu, s} l_{\nu, p s}\left(\frac{t}{h}\right) \quad q_{d}^{f}\left(t ; q_{k}^{f}, h\right)=\sum_{\nu=0}^{p f} q_{k}^{\nu, f} l_{\nu, p f}\left(\frac{t}{h}\right)
$$

Differentiating $q_{d}^{s}$ and $q_{d}^{f}$ w.r.t. $t$ provides approximations of $\dot{q}^{s}$ and $\dot{q}^{f}$ on $[0, h]$

$$
\dot{q}_{d}^{s}\left(t ; q_{k}^{s}, h\right)=\frac{1}{h} \sum_{\nu=0}^{p s} q_{k}^{\nu, s} \dot{l}_{\nu, p s}\left(\frac{t}{h}\right) \quad \dot{q}_{d}^{f}\left(t ; q_{k}^{f}, h\right)=\frac{1}{h} \sum_{\nu=0}^{p f} q_{k}^{\nu, f} \dot{l}_{\nu, p f}\left(\frac{t}{h}\right)
$$

Let the time interval $[0, T]$ be divided into $N=T / h$ subintervals of lengths $h$. On each subinterval, the continuous curves of the slow and fast configurations are approximated piecewisely via the polynomials $q_{d}^{s}:[k h,(k+1) h] \rightarrow Q^{s}$ and $q_{d}^{f}:[k h,(k+1) h] \rightarrow Q^{f}, k=0, \ldots, N-1$. To guarantee continuity on $[0, T]$ the conditions $q_{k}^{p s, s}=q_{k+1}^{0, s}$ and $q_{k}^{p f, f}=q_{k+1}^{0, f}$ for all $k=$ $0, \ldots, N-2$ must hold.

Replacing the continuous curves piecewisely by the corresponding polynomials and approximating the integral of the Lagrangian via appropriate quadrature formulas yield an approximation of the action $S$ on each subinterval $[k h,(k+1) h], k=0, \ldots, N-1$. In particular, different quadrature formulas for the approximation of the integral of the contributions of the Lagrangian are considered. The quadrature rule $\left(b_{i}^{t}, c_{i}^{t}\right)_{i=1}^{r^{t}}$ is chosen for the integral of the kinetic energy, $\left(b_{i}^{v}, c_{i}^{v}\right)_{i=1}^{r^{v}}$ for the integral of the slow potential and $\left(b_{i}^{w}, c_{i}^{w}\right)_{i=1}^{r^{w}}$ for the integral of the fast potential. The quadrature rules are all w.r.t. the time interval $[0,1]$ with quadrature points $c_{i}^{j} \in[0,1]$ and weights $b_{i}^{j} \in \mathbb{R} i=1, \ldots, r^{j}, j=v, w, t$. The discrete action then reads

$$
S_{d}\left(q_{0}, \ldots, q_{N-1}\right)=\sum_{k=0}^{N-1} L_{d}\left(q_{k}\right)
$$


with $q_{k}=\left(q_{k}^{s}, q_{k}^{f}\right)$ and the discrete Lagrangian

$$
\begin{aligned}
L_{d}\left(q_{k}\right) & =h \sum_{i=1}^{r^{t}} b_{i}^{t} T\left(\left(\dot{q}_{d}^{s}\left(c_{i}^{t} h ; q_{k}^{s}, h\right), \dot{q}_{d}^{f}\left(c_{i}^{t} h ; q_{k}^{f}, h\right)\right)\right) \\
& -h \sum_{i=1}^{r^{v}} b_{i}^{v} V\left(\left(q_{d}^{s}\left(c_{i}^{v} h ; q_{k}^{s}, h\right), q_{d}^{f}\left(c_{i}^{v} h ; q_{k}^{f}, h\right)\right)\right)-h \sum_{i=1}^{r^{w}} b_{i}^{w} W\left(\left(q_{d}^{f}\left(c_{i}^{w} h ; q_{k}^{f}, h\right)\right)\right)
\end{aligned}
$$

providing an approximation of the action (1). The discrete version of Hamilton's principle implies the discrete action $S_{d}$ to be stationary. Hence the derivatives of the discrete action $S_{d}$ w.r.t. $q_{k}^{\nu, s}, \nu=0, \ldots, p s$ and $q_{k}^{\nu, f}, \nu=0, \ldots, p f$ have to vanish $\forall k=0, \ldots, N-1$, with $\delta q_{0}^{0, s}=\delta q_{0}^{0, f}=\delta q_{N}^{0, s}=\delta q_{N}^{0, f}=0$. This leads to the discrete Euler-Lagrange equations

$$
\begin{aligned}
0= & D_{p s+1} L_{d}\left(q_{k}^{0, s}, \ldots, q_{k}^{p s, s}, q_{k}^{0, f}, \ldots, q_{k}^{p f, f}\right)+D_{1} L_{d}\left(q_{k+1}^{0, s}, \ldots, q_{k+1}^{p s, s}, q_{k+1}^{0, f}, \ldots, q_{k+1}^{p f, f}\right) \\
& k=0, \ldots, N-2 \\
0= & D_{i} L_{d}\left(q_{k}^{0, s}, \ldots, q_{k}^{p s, s}, q_{k}^{0, f}, \ldots, q_{k}^{p f, f}\right) \quad i=2, \ldots, p s \quad k=0, \ldots, N-1 \\
0= & D_{(p s+1)+p f+1} L_{d}\left(q_{k}^{0, s}, \ldots, q_{k}^{p s, s}, q_{k}^{0, f}, \ldots, q_{k}^{p f, f}\right) \\
& +D_{(p s+1)+1} L_{d}\left(q_{k+1}^{0, s}, \ldots, q_{k+1}^{p s, s}, q_{k+1}^{0, f}, \ldots, q_{k+1}^{p f, f}\right) \quad k=0, \ldots, N-2 \\
0= & D_{(p s+1)+i} L_{d}\left(q_{k}^{0, s}, \ldots, q_{k}^{p s, s}, q_{k}^{0, f}, \ldots, q_{k}^{p f, f}\right) \quad i=2, \ldots, p f \quad k=0, \ldots, N-1
\end{aligned}
$$

with $D_{\alpha}$ being the partial derivative of the discrete Lagrangian w.r.t. the $\alpha$-th argument. The discrete Legendre transforms $\mathbb{F}_{L_{d}}^{ \pm}: Q \times Q \rightarrow T^{*} Q$ read

$$
\begin{aligned}
& \mathbb{F}_{L_{d}}^{+}:\left(\begin{array}{c}
\left(q_{k-1}^{0, s}, q_{k-1}^{p s, s}\right) \mapsto\left(q_{k-1}^{p s, s}, p_{k-1}^{p s, s}\right)= \\
\left(q_{k-1}^{0, f}, q_{k-1}^{p f, f}\right) \mapsto\left(q_{k-1}^{p f, f}, p_{k-1}^{p f, f}\right)=\left(q_{k-1}^{p f, f}, D_{(p s+1)+p f+1} L_{d}\left(q_{k-1}\right)\right)
\end{array}\right) \\
& \mathbb{F}_{L_{d}}^{-}:\left(\begin{array}{c}
\left(q_{k}^{0, s}, q_{k}^{p s, s}\right) \mapsto\left(q_{k}^{0, s}, p_{k}^{0, s}\right)=\left(q_{k}^{0, s},-D_{1} L_{d}\left(q_{k}\right)\right) \\
\left(q_{k}^{0, f}, q_{k}^{p f, f}\right) \mapsto\left(q_{k}^{0, f}, p_{k}^{0, f}\right)=\left(q_{k}^{0, f},-D_{(p s+1)+1} L_{d}\left(q_{k}\right)\right)
\end{array}\right)
\end{aligned}
$$

Note that along the solution of the discrete Euler-Lagrange equations (2) it holds that $\left(p_{k-1}^{p s, s}, p_{k-1}^{p f, f}\right)=\left(p_{k}^{0, s}, p_{k}^{0, f}\right)=p_{k}^{0}$ and therefore the conjugate momentum $p_{k}^{0}$ is uniquely defined.

For given configurations $q_{k}=\left(q_{k}^{s}, q_{k}^{f}\right)=\left(q_{k}^{0, s}, \ldots, q_{k}^{p s, s}, q_{k}^{0, f}, \ldots, q_{k}^{p f, f}\right)$ the unknown configurations $q_{k+1}^{i, s}, i=1, \ldots, p s$ and $q_{k+1}^{i, f}, i=1, \ldots, p f$ in $q_{k+1}=\left(q_{k+1}^{s}, q_{k+1}^{f}\right)=\left(q_{k+1}^{0, s}, \ldots, q_{k+1}^{p s, s}\right.$, $\left.q_{k+1}^{0, f}, \ldots, q_{k+1}^{p f, f}\right)$ are obtained by solving the discrete Euler-Lagrange equations (2). Replacing the first and the third equation in (2) by the discrete Legendre transforms

$$
\begin{aligned}
& p_{0}^{0, s}=-D_{1} L_{d}\left(q_{0}\right) \\
& p_{0}^{0, f}=-D_{(p s+1)+1} L_{d}\left(q_{0}\right)
\end{aligned}
$$

provides a set of equations to determine the unknown configurations in $q_{0}$ in the first time step for given initial configuration $q(0)=q_{0}^{0}=\left(q_{0}^{0, s}, q_{0}^{0, f}\right)$ and momentum $p(0)=p_{0}^{0}=\left(p_{0}^{0, s}, p_{0}^{0, f}\right)$.

Varying the quadrature formulas (in this work the Gauss and the Lobatto quadrature are used) and its orders and varying the degrees $p s$ and $p f$ of the polynomials, provides a high number 
of possibilities to construct the integrators. The abbreviations ordT, ordV, ordW are introduced to denote the order of the quadrature formula that approximates the integral of the kinetic energy $T$, of the slow potential $V$ and of the fast potential $W$, respectively. Furthermore, if $\operatorname{ord} T=\operatorname{ord} V=\operatorname{ord} W$, the notation $\operatorname{ord} T V W$ is used, respectively if $\operatorname{ord} T=\operatorname{ord} W$ the notation $o r d T W$. Finally, an integrator is indicated as $\left[p s_{-} p f_{-}\right.$ord $d T_{-}(\mathrm{Gau} / \mathrm{Lob}) o r d V_{-}(\mathrm{Gau} / \mathrm{Lob})$ ord $\left.W_{-}(\mathrm{Gau} / \mathrm{Lob})\right]$ with the abbreviations Lob for Lobatto and Gau for Gauss quadrature.

\section{Examples}

- $[p s 2 p f 5$ ordTW $10(\mathrm{Gau})$ ord $V 4(\mathrm{Lob})]$ denotes the integrator that is obtained when the polynomial approximating the slow variables is of degree 2 , the polynomial approximating the fast variables is of degree 5. For the approximation of the integral of the kinetic energy $T$ and the fast potential $W$, the same quadrature formula is used, i.e. the Gauss quadrature of order 10, whereas the integral of the slow potential is approximated via the Lobatto quadrature of order 4.

- $[p s 1 p f 1$ ordTW2(Gau)ordV2(Lob)] yields an integrator treating the slow potential explicitly and the fast potential implicitly. This integrator is called the IMEX (implicitexplicit) method, introduced and analyzed in [10].

- Consider a linear polynomial $q_{d}^{s}$ and a polynomial $q_{d}^{f}$ of degree $p f$ to approximate the slow variable $q^{s}$ and the fast variable $q^{f}$, respectively. Suppose the kinetic energy $T$ and the fast potential $W$ are both approximated via the Gauss quadrature of order $2 p f$. For the approximation of the slow potential, different quadrature formulas are considered. Using the Lobatto quadrature of order 2 yields the integrator $\left[p s 1 p f \_o r d T W 12(\mathrm{Gau})\right.$ ordV2(Lob)] with the discrete slow potential

$$
V_{d}\left(q_{k}^{s}, q_{k}^{f}\right)=\frac{h}{2}\left[V\left(q_{k}^{0, s}, q_{k}^{0, f}\right)+V\left(q_{k}^{1, s}, q_{k}^{p f, f}\right)\right]
$$

The corresponding discrete conservative slow forces in the discrete Euler-Lagrange equations are explicit, i.e. they only depend on known configurations. When the Gauss quadrature of order 2 is applied, the integrator is [ps1pf_ordTW12(Gau)ordV2(Gau)]. The discrete slow potential then reads

$$
V_{d}\left(q_{k}^{s}, q_{k}^{f}\right)=h V\left(\frac{q_{k}^{0, s}+q_{k}^{1, s}}{2}, q_{d}^{f}\left(\frac{h}{2} ; q_{k}^{f}, h\right)\right)
$$

and the corresponding discrete conservative slow forces in the discrete Euler-Lagrange equations are implicit, meaning they depend on known and unknown configurations.

\section{NUMERICAL EXAMPLE}

\subsection{Fermi-Pasta-Ulam problem}

The performance of the variational integrators of mixed order is investigated numerically by means of the Fermi-Pasta-Ulam (FPU) problem (see e.g. [5]). The FPU model consists of $2 l$ unit masses that are chained together by alternating soft nonlinear and stiff linear springs with fixed end points. Appropriate coordinates are chosen that enable the separation of the multirate system in slow and fast variables. The scaled displacement of the $i$-th stiff spring's 
center is described by the slow variables $q_{i}^{s}, i=1, \ldots, l$, while the fast variables $q_{i}^{f}, i=1, \ldots, l$ represent the scaled length of the $i$-th stiff spring. The Lagrangian reads

$$
\begin{aligned}
L(q, \dot{q})= & \frac{1}{2} \sum_{i=1}^{l}\left(\left(\dot{q}_{i}^{s}\right)^{2}+\left(\dot{q}_{i}^{f}\right)^{2}\right)+ \\
& -\frac{1}{4}\left[\left(q_{1}^{s}-q_{1}^{f}\right)^{4}+\sum_{i=1}^{l-1}\left(q_{i+1}^{s}-q_{i+1}^{f}-q_{i}^{s}-q_{i}^{f}\right)^{4}+\left(q_{l}^{s}+q_{l}^{f}\right)^{4}\right]-\frac{\omega^{2}}{2} \sum_{i=1}^{l}\left(q_{i}^{f}\right)^{2}
\end{aligned}
$$

The first two terms in the Lagrangian, the kinetic energy and the slow potential $V$, are both depending on the complete configuration variable, whereas the fast potential $W$, i.e. the third term, depends on the fast variable only. The stiffness $\omega \in \mathbb{R}$ in $W$ is supposed to be large. The FPU problem is a multirate system because it shows different behaviour on different time scales [5]. The vibration of the stiff springs happens on the time scale $\omega^{-1}$. On the time scale $\omega^{0}$, the motion of the soft nonlinear springs takes place and over intervals exponentially long in $\omega$, an energy exchange among the stiff springs occurs. For the simulations, the FPU model consists of 6 point masses, i.e. $l=3$, with mass $m=1$ and the stiffness of the stiff springs is $\omega=50$. All initial values are zero despite the displacement and the extension of the first stiff spring and their velocities with $q_{1}^{s}(0)=1, q_{1}^{f}(0)=\omega^{-1}, \dot{q}_{1}^{s}(0)=1, \dot{q}_{1}^{f}(0)=1$.

The performance of the variational integrators of mixed order is demonstrated by means of the motion of the first stiff spring and the energy exchange between the stiff springs over a long term simulation. The solution of the standard variational integrator [ps $1 p f 1$ ordTVW $2(\mathrm{Gau})]$, i.e. the midpoint-rule, with time step $h=10^{-5}$ serves as a reference. Moreover, a numerical analysis regarding efficiency versus accuracy is carried out.

In Fig. 1, the motion of the first stiff spring's center $q_{1}^{s}$ and the oscillation of the first stiff spring's extensions $q_{1}^{f}$ are shown. The reference solutions are always plotted in solid turquoise. In the left hand side plots in Fig. 1, one can see in purple dashed the simulation results of the standard variational integrator $[p s 1 p f 1 \operatorname{ord} T V W 2(\mathrm{Gau})]$ with time step $h=0.1$. Increasing the degree of the polynomial for the fast part from one to six, while the approximation of the slow variable is still linear and using a quadrature formula of order 12 for $T$ and $W$, yields the integrator $[p s 1 p f 6$ ordTW12(Gau)ordV2(Gau)]. Using the time step $h=0.1$, the right hand side of Fig. 1 shows its discrete solution for the slow and fast configuration in purple dashed. With $h=0.1$, both integrators resolve the motion of the slow variable $q_{1}^{s}$ quite well, see top plots in Fig. 1 a) and b). The high degree of 6 of the polynomial approximating the fast configuration causes that the simulated fast motion nicely coincides with the reference solution, cf. lower plot in Fig. 1 b). Whereas, when using the linear polynomial for the fast part, the fast motion is not captured as can be seen in the lower plot in Fig. 1 a). Note that the integrator $[p s 1 p f 6$ ordTW $12(\mathrm{Gau}) \operatorname{ord} V 2(\mathrm{Gau})]$ provides the fast configurations $q_{k}^{f}=\left(q_{k}^{0, f}, \ldots, q_{k}^{6, f}\right)$ for one time step $[k h,(k+1) h]$ such that with equidistant control points $d_{\nu}^{f}, \nu=0, \ldots, 6$ the configurations $q_{k}^{i, f}$ at the time points $k h+i h / 6, i=0, \ldots, 6$ are determined. Moreover, to resolve the high oscillations of the first stiff spring with a standard variational integrator, a smaller time step is needed. Compare Fig. 1 c) where the fast configuration $q^{f}$ simulated via the standard variational integrator $[p s 1 p f 1$ ordTVW $2(\mathrm{Gau})]$ with time step $h=0.01$ is plotted. The high oscillation of $q_{1}^{f}$ is captured, however the solution is shifted compared to the reference solution as typically seen for the standard variational integrators. This shifting-effect amplifies with increasing simulation time. No phase shifting occurs in the lower plot of Fig. 1 b), where the simulation results of the integrator $[$ ps1pf6ordTW12(Gau)ordV2(Gau)] are depicted, even after a long simulation time e.g. of 30 seconds (Fig. 1 d)). 
The energies of the stiff springs (red/purple/green) exchange over the time, while the sum of the stiff springs' energy (blue) remains close to a constant value, which is called an adiabatic invariant of the Hamiltonian system [5]. Fig. 2 is the reference solution. The plots in Fig. 3 show the simulation results of the integrators $[\operatorname{ps} 1 p f 6$ ordTW12(Gau) ordV2(Gau)], $[p s 1 p f 6$ ordTW12(Gau)ordV2(Lob)] and [ps1pf6ordTVW12(Gau)]. In the left hand side plots of Fig. 3, the time step $h=0.1$ is used for the simulations. The use of a quadrature formula of order 2 for the approximation of the slow potential, regardless of being the Gauss or the Lobatto quadrature, leads to high deviations in the oscillatory energies and their sum (Fig. 3 a) c)). However, both methods give qualitatively the correct rate of energy exchange and the total energy shows oscillations but no numerical drift. Increasing the order of $\operatorname{ord} V$ to 12 , the deviations of the oscillatory energies decrease and have the same magnitude as in the reference solution. The exchange of the stiff springs' energies is captured and the total oscillatory energy is nearly constant, see Fig. 3 e). Choosing $h=0.2$ as time step, the performance of the integrators degrades, but the qualitative structure of the energy exchange behaviour is still maintained, see Fig. 3 b) d) f).

The computational efficiency of the variational integrators of mixed order is investigated numerically. The plots in Fig. 4 show the global errors of the slow configuration $q^{s}$ (left) and of the fast configuration $q^{f}$ (right) for the time steps $[0.2,0.1,0.05,0.01,0.005]$ against the run-times. The global errors of the configurations are determined by

$$
\max _{k \in\{0, \ldots, N\}}\left\|q_{k}^{0, s}-q_{r e f}^{s}(k h)\right\| \quad \max _{k \in\{0, \ldots, N\}}\left\|q_{k}^{0, f}-q_{r e f}^{f}(k h)\right\|
$$

where $q_{r e f}^{s}$ and $q_{r e f}^{f}$ are the configurations of the reference solution. Due to the limited accuracy of the reference solution itself, the global errors of the slow and the fast configuration can only be determined to a magnitude of approximately $10^{-8}$ and $10^{-7}$, respectively. The simulation time is $T=6$ seconds. Starting with the integrator $[p s 6 p f 6 o r d T V W 12(\mathrm{Gau})]$ (blue dashed) in Fig. 4 a), the degree of the polynomial $q_{d}^{s}$ approximating the slow variable is decreased successively from 6 to 1 , while the degree of $q_{d}^{f}$ is still 6 and the orders of the quadrature formulas remain 12. Reducing the degree $p s$ of $q_{d}^{s}$ decreases the number of unknowns in the EulerLagrange equations and thus the run-time decreases. Though, the accuracy of the solutions of the integrators with a lower degree $p s$ remains nearly the same, which is nicely visible in both plots in Fig. 4 a) for the polynomial degrees $p s=3,4$ and 5. This result demonstrates the improved efficiency of the proposed mixed order integrators. However, decreasing $p s$ even more causes a loss of accuracy of the simulation results, which becomes obvious for the degrees $p s=1$ and $p s=2$ in Fig. 4 a). To assess the computational efficiency of the integrators, the desired accuracy is crucial. For instance, when the focus lies on the fast configuration only with a lower accuracy being sufficient, the integrator $[p s 1 p f 6$ ordTVW12(Gau)] (red dashed) is the fastest in our implementation, see right plot in Fig. 4 a). More accurate results of the fast and the slow configuration in conjunction with shortest run-times are achieved by the integrator $[p s 3 p f 6$ ordTVW12(Gau)] (green dashed) in Fig. 4 a). Further savings in run-time can be reached, when the order $\operatorname{ord} V$ decreases, as a low order ord $V$ means less quadrature nodes and therefore less costly function evaluations of the non-linear slow potential. Thus, successively reducing the order $\operatorname{ord} V$ of the integrator $[\operatorname{ps} 1 p f 6 \operatorname{ordTV} W 12(\mathrm{Gau})]$ (Fig. $4 \mathrm{~b})$ ), respectively [ps3pf6ordTVW12(Gau)] (Fig. 4 c)), shortens the run-time even more with negligible influence on the accuracy, see Fig. 4 b) and c), where the errors for $\operatorname{ord} V=6,8,10,12$ for $p s=1$ ( $\operatorname{ord} V=8,10,12$ for $p s=3$, respectively) are of the same magnitude. However, decreasing the order $\operatorname{ord} V$ too far leads to loss of accuracy in the configuration. First investigations indi- 
cate that the loss is a result of convergence order reductions.

Fig. 5 shows the savings in run-time compared to [ps6pf6ordTVW12(Gau)] at a glance. If a lower accuracy of the fast configuration is sufficient, a decreased degree $p s$ of 1 (red dashed) and in addition a low quadrature order $\operatorname{ord} V=4$ (yellow dash-dotted) bring shortest run-times in our implementation. A more accurate result can be gained cheaply with a decreased degree $p s=$ 3 (green dashed) and most cheaply together with order $\operatorname{ord} V=8$ (purple dash-dotted). Furthermore, the run-time compared with the global error of $q^{f}$ for the integrators $[p s 1 p f 6 o r d T W 12$ (Gau)ordV2 (Gau)] (blue dash-dotted) and [ps1pf6ordTW12 (Gau)ordV2 (Lob)] (blue dotted) and for the standard variational integrator $[p s 1 p f 1$ ordTWV2(Gau)] (turquoise dashed) are plotted in Fig. 5. It is nicely visible that treating the slow force explicitly (blue dotted) saves run-time compared to the implicit treatment of the slow force (blue dash-dotted). In comparison to the standard variational integrator, great improvements regarding efficiency versus accuracy are achieved with the presented integrators. The global error of the fast configuration simulated with the standard variational integrator $[p s 1 p f 1$ ordTWV $2(\mathrm{Gau})]$ does not get smaller for the tested time steps between $h=0.2$ and $h=0.01$. The reason is the phase shifting that proceeds so far that the reference solution and the solution of the standard variational integrator are phase shifted by half a period causing a global error of two times the amplitude of the first spring's oscillation. Hence, the global error of the standard variational integrator [ps1pf1ordTWV2(Gau)] must be regarded in relation to the simulation time, as the shiftingeffect amplifies with it. 

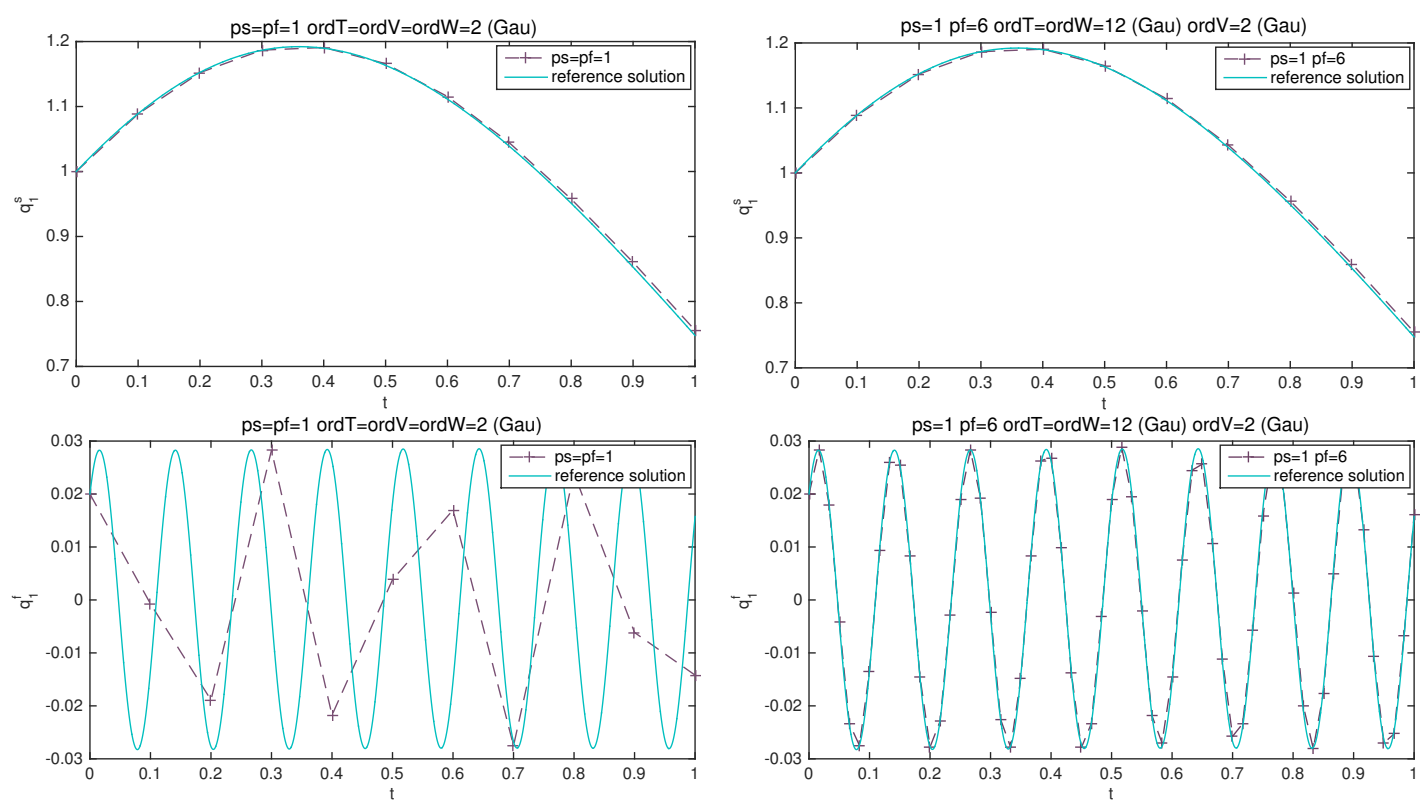

a) $h=0.1$

b) $h=0.1$
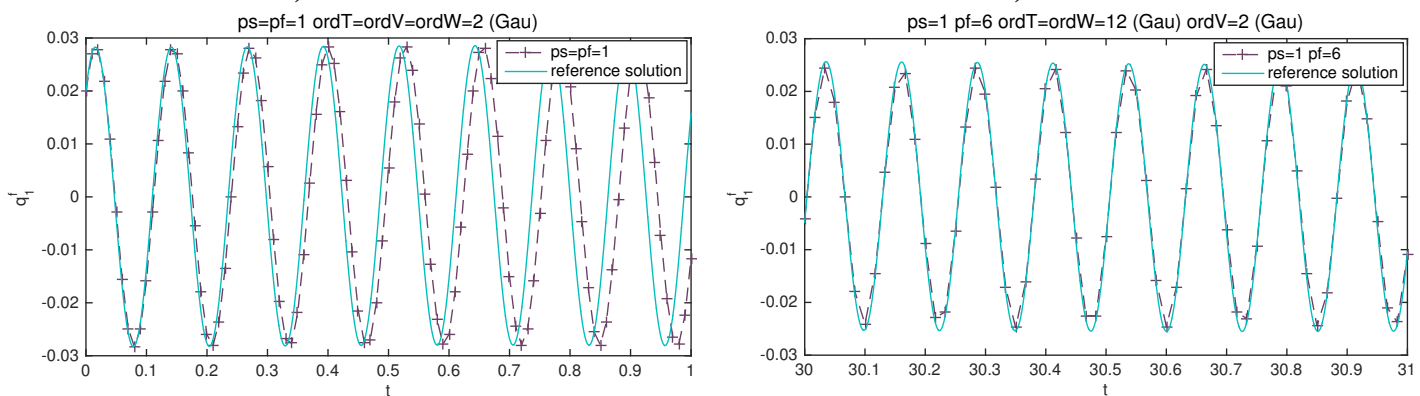

c) $h=0.01$

d) $h=0.1$

Figure 1: FPU problem. Simulation results of the first slow $q_{1}^{s}$ and first fast $q_{1}^{f}$ configuration using a standard variational integrator (left) with time step $h=0.1$ in a) and $h=0.01$ in c) and using the integrator $[p s 1 p f 6$ ordTW12(Gau)ordV2(Gau)] (right) with time step $h=0.1$ in b) and d) in dashed purple, reference solution in solid turquoise.

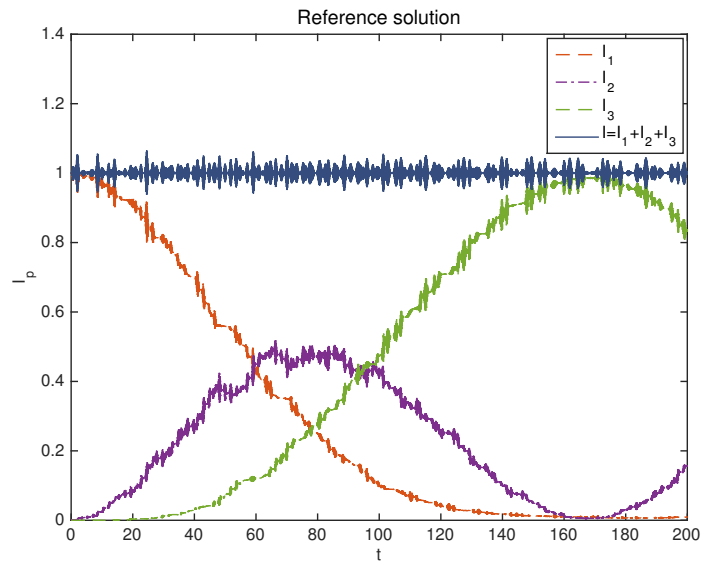

Figure 2: FPU problem. Energy of the three stiff springs (red dashed, purple dash-dotted, green dashed) and the total oscillatory energy (blue solid) of the reference solution. 


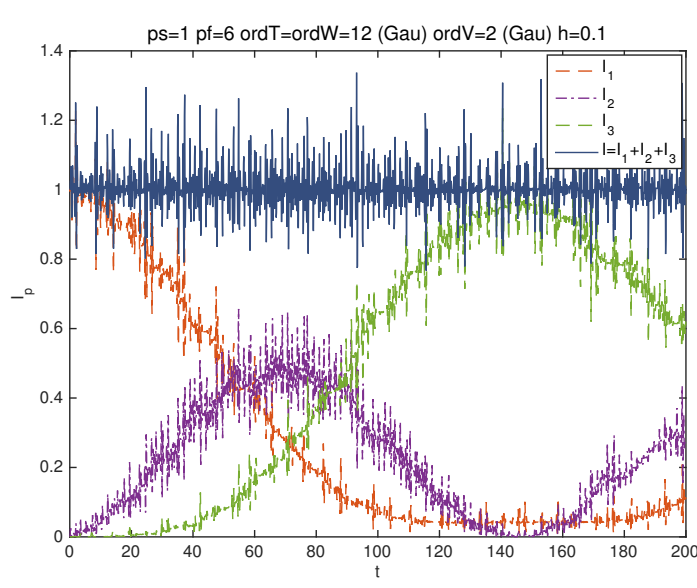

a)

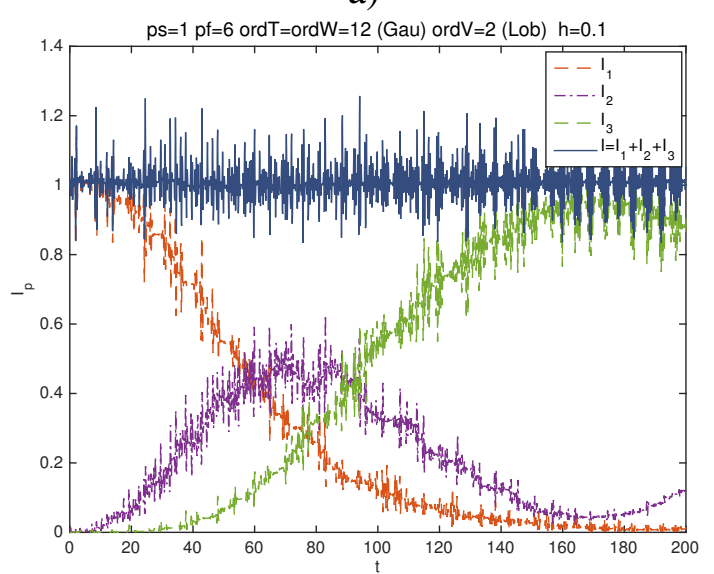

c)

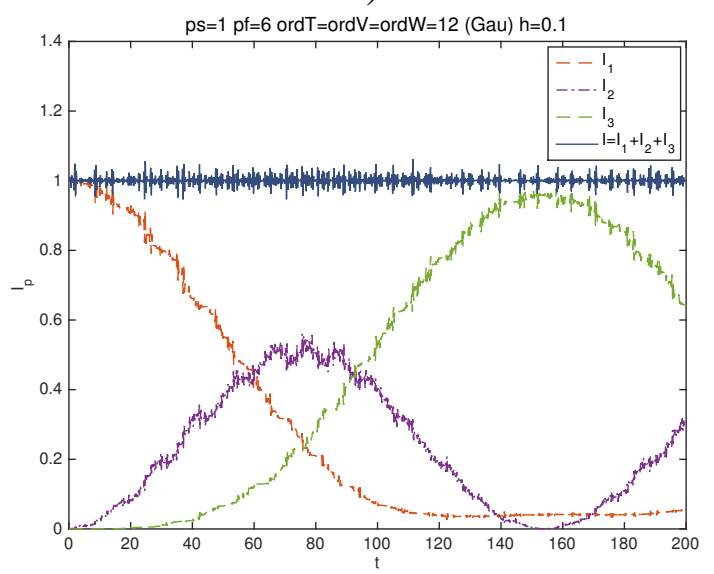

e)

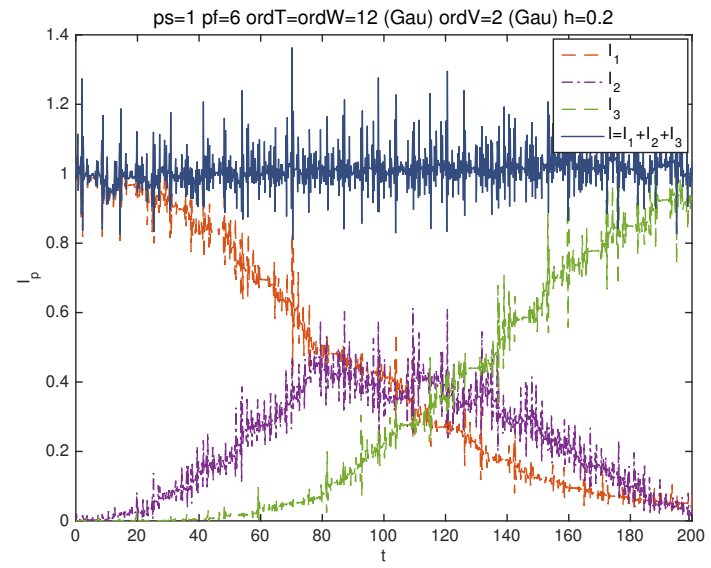

b)

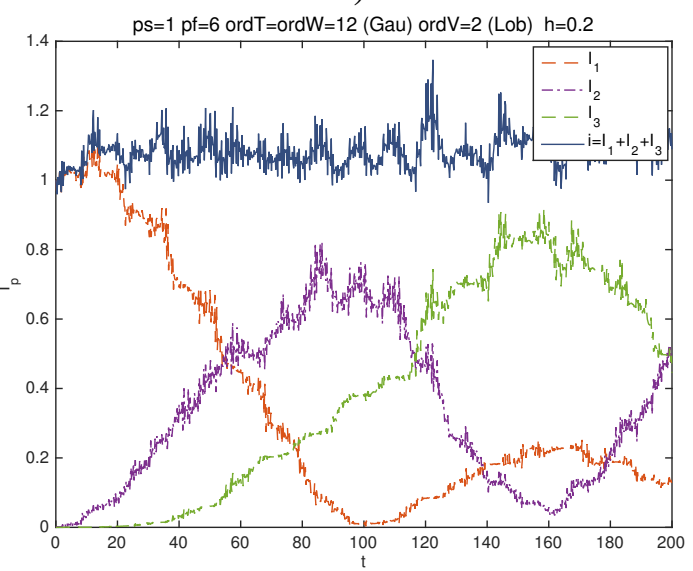

d)

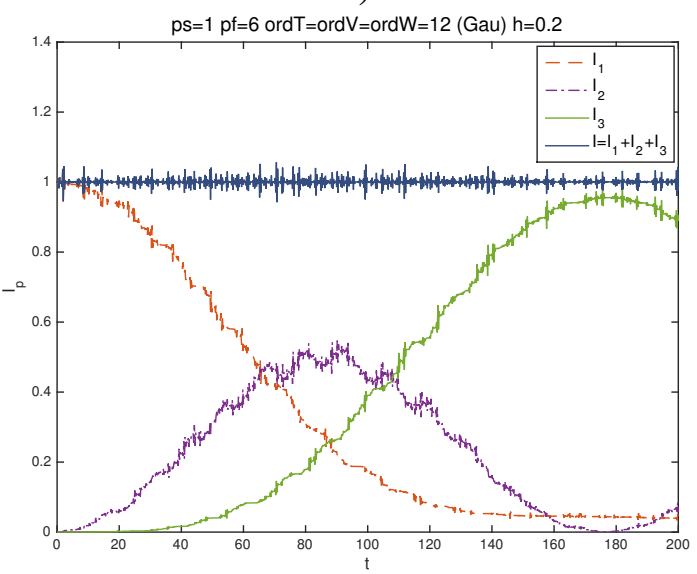

f)

Figure 3: FPU problem. Energy of the three stiff springs (red dashed, purple dashdotted, green dashed) and the total oscillatory energy (blue solid). Simulation results of the integrators [ps1pf6ordTW12(Gau)ordV2(Gau)], [ps1pf6ordTW12(Gau)ordV2(Lob)] and $[\operatorname{ps} 1 p f 6$ ordTVW12(Gau)] with time step $h=0.1$ (left) and $h=0.2$ (right). 

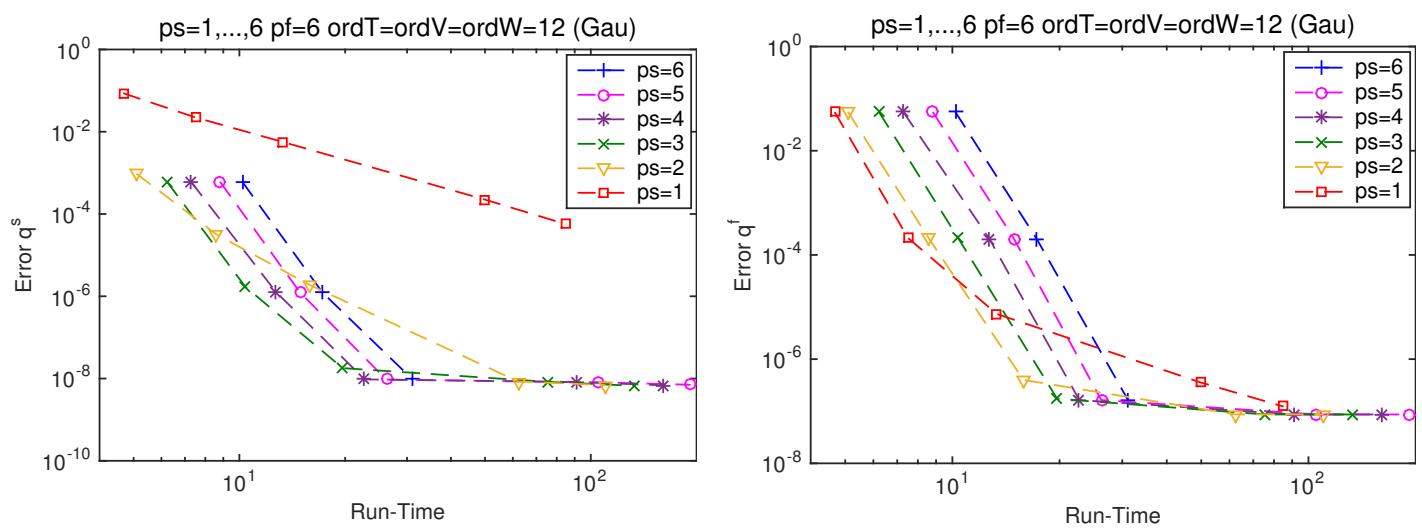

a)
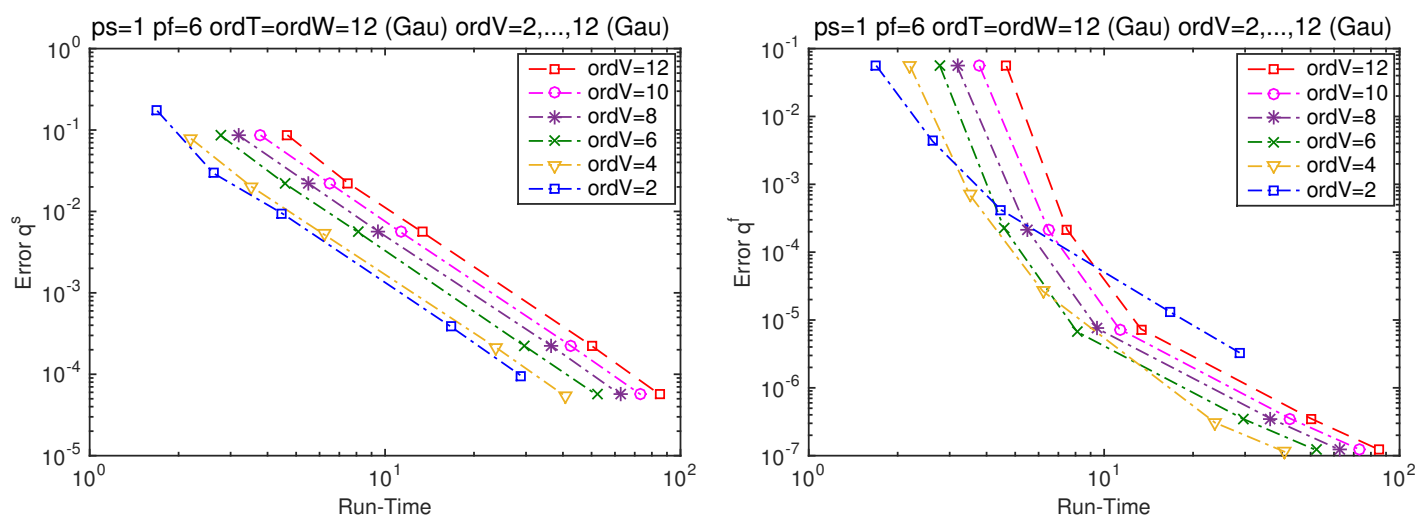

b)
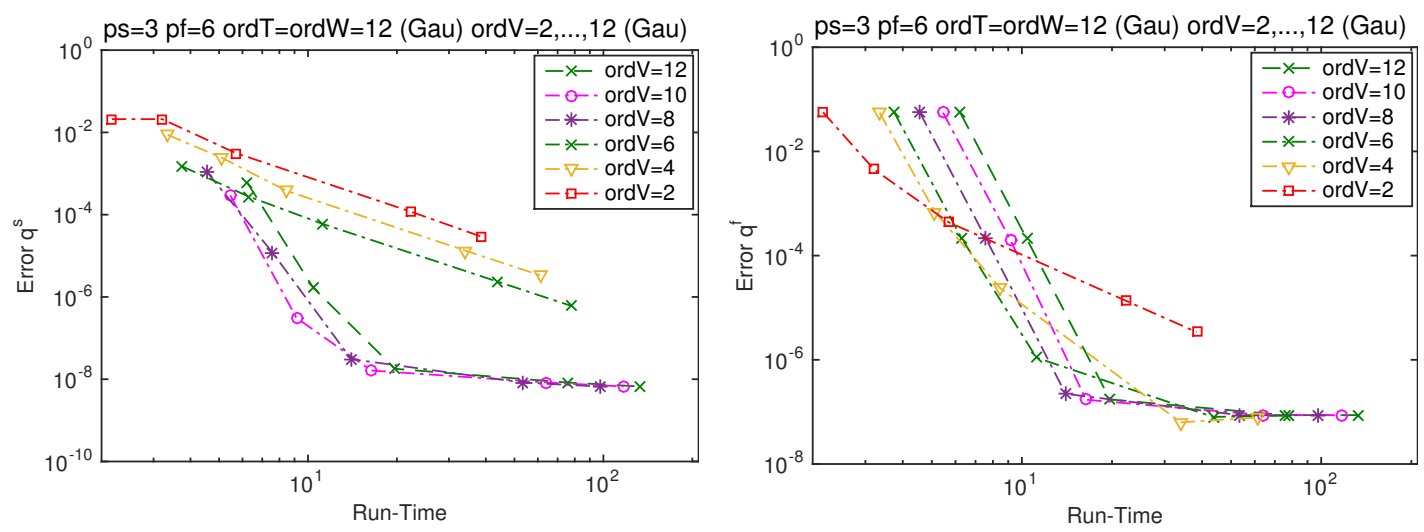

c)

Figure 4: FPU problem. Global error of the slow configuration $q^{s}$ (left) and the fast configuration $q^{f}$ (right) against run-time for the simulation time of $T=6$ seconds for the integrators $[p s 1 \ldots 6 p f 6 o r d T V W 12(\mathrm{Gau})]$ in a), [ps1pf6ordTW12(Gau)ordV2 .. 12(Gau)] in b) and $[p s 3 p f 6 o r d T W 12(\mathrm{Gau}) \operatorname{ord} V 2 \ldots 12(\mathrm{Gau})]$ in c) with time steps sizes $h \in\{0.2,0.1,0.05,0.01,0.005\}$. 


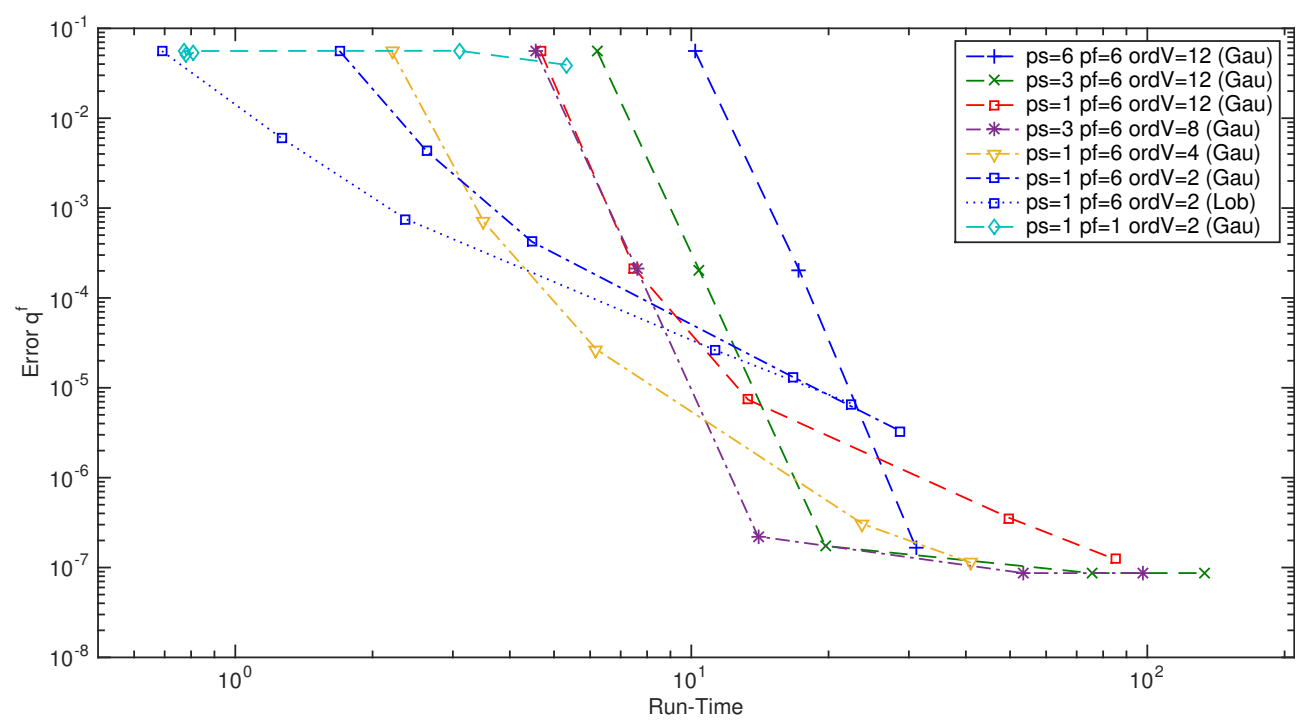

Figure 5: FPU problem. Global error of the fast configuration against run-time for the simulation time of $T=6$ seconds for different integrators [ps_pf_ordTW12(Gau)ordV_(Gau / Lob)] with time steps sizes $h \in\{0.2,0.1,0.05,0.01,0.005\}$.

\section{CONCLUSION}

The integrators of mixed order feature a great flexibility in design and thus in application. In this work, particular attention is paid to mechanical systems that act on different time scales caused by slow nonlinear and fast quadratic potentials. The trade-off between high accuracy and low computational costs, when simulating such mechanical systems, is resolved via a low degree polynomial for approximating the slow and a high degree polynomial for approximating the fast motion. The degrees of the polynomials have a direct impact on the number of unknowns in one time step and thus on run-time. Furthermore, due to its approximation via a high degree polynomial, the fast motion is captured and the typical phase shifting observed for the standard variational integrator is prevented. Considering the approximation of the integrals of the contributions of the Lagrangian separately enables the use of different quadrature formulas for each part and especially a low order quadrature formula for the integral of the slow potential reduces the number of costly function evaluations. This approach offers a possibility to decrease run-time with negligible loss in accuracy at once. However, higher savings in runtime come at a cost, as the accuracy suffers then. Therefore, the computational efficiency of the integrators has to be assessed with regard to the desired accuracy. The numerical investigation reveals that the multiscale phenomena of slow energy exchange and the conservation of adiabatic invariants are captured. However, the order of the quadrature formula approximating the integral of the slow energy influences the magnitude of the deviations of the oscillatory energies such that with increasing the order, the deviations decrease. The presented integrators base on a discrete version of Hamilton's principle. A detailed analysis of the inherited properties such as symplecticity and momentum preservation has to be done in the future, as well as a stability analysis. The convergence order of the integrators of mixed order are of interest, inter alia with regard to the observed losses in accuracy. Furthermore, a next step is to extend the approach to mechanical systems acting on multiple time scales with holonomic constraints. A comparison of the multirate variational integrators based on multiple time grids to the approach presented here is also subject of future works. 


\section{REFERENCES}

[1] M. Arnold, Multi-rate time integration for large scale multibody system models. Proceedings of the IUTAM Symposium on Multiscale Problems in Multibody System Contacts, Stuttgart, Germany, February 20-23, 2006.

[2] C. Engstler and C. Lubich, Multirate extrapolation methods for differential equations with different time scales. Computing, 58, 173-185, 1997.

[3] C. W. Gear and D. R. Wells, Multirate linear multistep methods. BIT Numerical Mathematics, 24, 484-502, 1984.

[4] M. Günther, A. Kvaernø, and P. Rentrop, Multirate partitioned Runge-Kutta methods. BIT Numerical Mathematics, 41, 504-514, 2001.

[5] E. Hairer, C. Lubich, and G. Wanner, Geometric Numerical Integration: StructurePreserving Algorithms for Ordinary Differential Equations, Vol. 31 of Springer Series in Computational Mathematics. Springer, Berlin Heidelberg, 2006.

[6] B. Leimkuhler and S. Reich, Simulating Hamiltonian dynamics, Vol. 14. Cambridge University Press, 2004.

[7] S. Leyendecker and S. Ober-Blöbaum, A variational approach to multirate integration for constrained systems. In Multibody dynamics, 97-121, Springer, 2013.

[8] J. E. Marsden and M. West, Discrete mechanics and variational integrators. Acta Numerica, 10, 357-514, 2001.

[9] S. Ober-Blöbaum and N. Saake, Construction and analysis of higher order Galerkin variational integrators. Advances in Computational Mathematics, 41, 955-986, 2015.

[10] A. Stern and E. Grinspun, Implicit-explicit variational integration of highly oscillatory problems. SIAM Multiscale Modeling \& Simulation, 7, 1779-1794, 2009.

[11] M. Tao, H. Owhadi, and J. E. Marsden, Nonintrusive and structure preserving multiscale integration of stiff ODEs, SDEs, and Hamiltonian systems with hidden slow dynamics via flow averaging. SIAM Multiscale Modeling \& Simulation, 8, 1269-1324, 2010.

[12] E. Weinan, B. Engquist, X. Li, W. Ren, and E. Vanden-Eijnden, Heterogeneous multiscale methods: a review. Communications in Computational Physics, 2, 367-450, 2007. 